\title{
Fisioterapia para Redução da Diástase dos Músculos Retos Abdominais no Pós-Parto
}

Physiotherapy for Reduction of Diastasis of the Recti Abdominis Muscles in the Postpartum Period

Luciana Aparecida Mesquita, Antônio Vieira Machado, Angela Viegas Andrade

\section{RESUMO}

Objetivos: constatar se a intervenção fisioterápica no puerpério imediato é capaz de contribuir para a redução da diástase mais precocemente.

Métodos: foi realizado um estudo longitudinal e aleatório, com 50 puérperas recrutadas na Maternidade-Escola Hilda Brandão da Santa Casa de Belo Horizonte (MEHB-SCBH), no periodo de abril a setembro de 1998. O grupo controle $(N=25)$ foi submetido à avaliação $e$ mensuração da diástase (6 horas e 18 horas após o parto) e no grupo de tratamento $(N=25)$ foi realizada a mesma avaliação e mensuração acima, assim como um protocolo de atendimento fisioterápico, 6 e 18 horas após o parto. Utilizou-se um paquimetro, instrumento de medida de precisão, na mensuração da diástase nas avaliações.

Resultados: no periodo de 18 horas pós-parto, o grupo controle apresentou uma redução da diástase de 5,4\% e o grupo de tratamento de 12,5\%, em relação à primeira medida (6 horas após o parto), ( $p<0,001$, com intervalo de confiança de 99\%).

Conclusões: estes resultados demonstram que o atendimento fisioterápico no puerpério imediato determina redução significativa na diástase dos músculos retos abdominais (DMRA) após cada atendimento, bem como redução relevante quando comparada com o grupo controle, contribuindo positivamente para a recuperação dos músculos retos abdominais mais precocemente.

PALAVRAS-CHAVE: Diástase dos músculos retos abdominais. Fisioterapia. Puerpério. Gravidez normal.

\section{Introdução}

O puerpério é um período de grandes modificações corporais e psíquicas, predominando um catabolismo intenso sem conseqüências patológicas, na maioria das vezes ${ }^{4}$. Como tem sido demonstrado e salientado na literatura ${ }^{6,15}$, é necessário que a puérpera seja assistida por uma equipe multidisciplinar, a fim de proporcionar-lhe

Maternidade-Escola Hilda Brandão da Santa Casa de Belo Horizonte.

Correspondência:

Luciana Aparecida Mesquita

Rua Vila Rica, 451/ Ap.302 - Bairro Padre Eustáquio

30.720-380 - Belo Horizonte - M.G.

e-mail: gom@gold.com.br segurança e conforto no puerpério imediato. Um dos objetivos da fisioterapia aplicada a esta etapa é promover uma estimulação da musculatura, em particular abdominal e pélvica, para melhorar a sua tonicidade ${ }^{13}$. Um programa de exercícios individuais e adaptados para cada paciente no período pós-parto tardio é importante para a recuperação da puérpera ${ }^{6}$. No entanto, percebe-se que esse atendimento não é comumente encontrado na rotina hospitalar da maioria das maternidades.

$\mathrm{Na}$ Maternidade-Escola Hilda Brandão da Santa Casa de Belo Horizonte (MEHB-SCBH) é realizado rotineiramente o atendimento fisioterápico no pós-parto imediato, por meio de um protocolo padronizado. No entanto, neste 
protocolo de atendimento, a mensuração da diástase dos músculos retos abdominais (DMRA) tem sido realizada de maneira subjetiva, ou seja, pela medida do número de dedos que podem ser inseridos entre os feixes musculares dos músculos retos abdominais, assim como ocorre nos demais estudos $^{2,3,5}$.

Durante a gestação, o estiramento da musculatura abdominal é indispensável para permitir o crescimento uterino, ocorrendo, portanto, uma separação dos feixes dos músculos retos abdominais ${ }^{13,17}$. Esta DMRA não provoca desconforto nem dor, apresentando incidência menor em mulheres com bom tônus abdominal antes da gravide $z^{8}$. A incidência, duração e complicação a curto e longo prazo da DMRA na gravidez não têm sido bem investigadas ${ }^{3}$. São considerados fatores predisponentes para a DMRA: obesidade, gestações múltiplas, multiparidade, poliidrâmnio, macrossomia fetal e flacidez da musculatura abdominal pré-gravídica, por levar a uma maior distensão abdominal durante a gravide $z^{3,16}$.

Noble $^{12}$ considera uma DMRA de dois ou menos dedos $( \pm 3 \mathrm{~cm})$ como normal nas regiões supra, infra e umbilical. Com este grau de diástase há retorno espontâneo às condições pré-gravídicas, sem complicações.

A incidência da DMRA é maior no terceiro trimestre da gestação e no pós-parto imediato ${ }^{2,3,5}$. É percebida inicialmente no segundo trimestre da gestação, apresentando diminuição no pós-parto tardio, porém não desaparecendo completamente até um ano após o parto ${ }^{2}$. A DMRA supra-umbilical é a mais significativa e a mais freqüente ${ }^{3,11}$.

Com o objetivo de guiar a palpação da DMRA Bursh $^{3}$ criou um dispositivo semelhante a uma régua de "Polyform". Este dispositivo era inserido no centro do umbigo, estendendo-se $4,5 \mathrm{~cm}$ acima e abaixo da cicatriz umbilical, tendo padronizado assim o local exato da verificação e medida da DMRA.

Durante a gestação, há uma anteversão pélvica acompanhada ou não de uma hiperlordose lombar ${ }^{15}$. Na maioria das gestantes há uma tendência de horizontalização do osso sacro. Essas alterações determinarão uma mudança do ângulo de inserção dos músculos abdominais e pélvicos ${ }^{1}$, resultando numa distensão excessiva dos mesmos, com conseqüente prejuízo do vetor de força destes músculos, com uma diminuição na força de contração? .

Essas alterações biomecânicas observadas no puerpério são decorrentes das modificações físicas pertinentes à gravide ${ }^{13}$. Após o parto, inicia-se um processo lento de reversão, que dura em média seis semanas, podendo se arrastar até três meses pós- parto $^{1}$. Assim, percebe-se a necessidade de exercícios físicos no pós-parto, desde que sejam supervisionados e direcionados por profissionais especializados, objetivando acelerar o processo de retorno às condições pré-gravídicas ${ }^{1,3}$.

Em estudos recentes observou-se a necessidade de um programa de exercícios para resolução mais breve da DMRA no pós-parto tardio e remoto ${ }^{1}$, a partir da comparação entre um grupo que realizava atividades fisicas controladas durante a gestação e pós-parto (grupo de tratamento) e outro grupo, controle. No grupo controle foi observada uma grande incidência de DMRA superior a $3 \mathrm{~cm}$, havendo uma demora da resolução espontânea desta condição, com influência na biomecânica postural e déficit na função de sustentação dos órgãos pélvicos e abdominais ${ }^{1}$.

A análise eletromiográfica da musculatura abdominal demonstrou que os exercícios abdominais estimulam a ação voluntária destes músculos ${ }^{4,8}$. Assim, o estímulo nesta região tende a melhorar a tonicidade da musculatura que se encontra flácida ou hipotônica ${ }^{14}$.

A atuação da fisioterapia no pós-parto imediato visa melhorar a tonicidade dos músculos abdominais e pélvicos, conscientizar as puérperas sobre a importância da continuidade dos exercícios iniciados neste período e sobre o retorno para o atendimento no pós-parto tardio.

$\mathrm{Na}$ maioria dos estudos ${ }^{2,3,5}$ foram utilizados parâmetros subjetivos (número de dedos) e não reprodutiveis para a mensuração da DMRA, mas um instrumento confiável, prático e de fácil manejo é necessário para tal ${ }^{3}$. Cientes da inexistência de pesquisas que relatassem o uso de instrumentos de medidas precisas na mensuração dessa diástase, surgiu então a necessidade de realizar um estudo que objetivasse medi-la com precisão e obter parâmetros fidedignos e reprodutíveis. Nesta pesquisa com caráter experimental e inovador, utilizou-se um medidor preciso de diâmetros e espessuras, o paquímetro, de uso comum na Engenharia, para a mensuração da DMRA supraumbilical. É importante ressaltar que o paquímetro obedece ao padrão internacional de medidas, oferecendo suas variáveis em milímetros ou polegadas.

Apesar de alguns estudos $3,5,6,15$ terem observado efeitos favoráveis do exercício no período pós-natal, nota-se que são raros os estudos que abordam o emprego do exercício no puerpério imediato. É fundamental comprovar que o atendimento fisioterápico no puerpério imediato pode contribuir para a redução precoce da DMRA. Portanto, este estudo se destinou a verificar se a intervenção fisioterápica no pós-parto imediato pode contribuir na redução desta diástase. 


\section{Pacientes e Métodos}

A pesquisa foi realizada na $\mathrm{MEHB}-\mathrm{SCBH}$, no período de abril a setembro de 1998, com a aprovação do Conselho de Ética desta instituição e com o consentimento das puérperas após esclarecimentos sobre a pesquisa.

A amostra foi constituída de 50 puérperas, recrutadas nas enfermarias de pós-parto da MEHB. A coleta de dados foi feita por amostragem aleatória, sendo que toda gestante que chegasse à maternidade em trabalho de parto poderia, teoricamente, participar da amostra, desde que preenchesse os critérios de inclusão. A amostra foi dividida aleatoriamente em dois grupos: um de controle e outro tratamento, com 25 puérperas cada um.

Foram incluídas no estudo as puérperas com idade entre 18 e 40 anos, hígidas, com até 4 filhos, que tiveram parto transvaginal sem anestesia de bloqueio epidural ou subdural. Deveriam apresentar DMRA superior a $3 \mathrm{~cm}$ e máximo de 6 horas após o parto.

Os critérios de exclusão foram: puérperas submetidas a parto cesariana; parto múltiplo; idade inferior a 18 anos ou superior a 40 anos; presença de quaisquer patologias associadas à gravidez (poliidrâmnio, macrossomia fetal, hipertensão arterial induzida pela gravidez (HAIG)), previamente diagnosticadas pelo corpo clínico da Maternidade, e uma DMRA inferior a $3 \mathrm{~cm}$.

Dados sobre cor, profissão e peso não foram critérios de inclusão e exclusão para esta pesquisa.

Para a mensuração da DMRA foi utilizado um paquímetro da marca NORFOL, conferindo exatidão e fidedignidade à coleta de dados. Foi utilizado um único paquímetro na mensuração da DMRA durante toda a pesquisa.

Toda coleta de dados e aplicação do protocolo experimental foi realizado por uma única pesquisadora.

A avaliação fisioterápica constou da observação do estado geral da paciente, para certificar-se da ausência de patologias ou instabilidades dos dados vitais associados ao puerpério, bem como da involução uterina e a mensuração da DMRA. Visou, portanto, analisar o aparelho respiratório, circulatório, o tônus da musculatura abdominal e a presença da DMRA.

Para a verificação da presença de DMRA a puérpera foi posicionada em decúbito dorsal, com o quadril e joelhos fletidos e com os pés apoiados no leito. A pesquisadora demarcou dois pontos: $4,5 \mathrm{~cm}$ acima e abaixo da cicatriz umbilical. A puérpera fez uma flexão anterior de tronco até que as bordas inferiores das escápulas saíssem do colchão. Neste momento, a pesquisadora fez a verificação e mensuração da DMRA com o paquímetro (Figura 1).

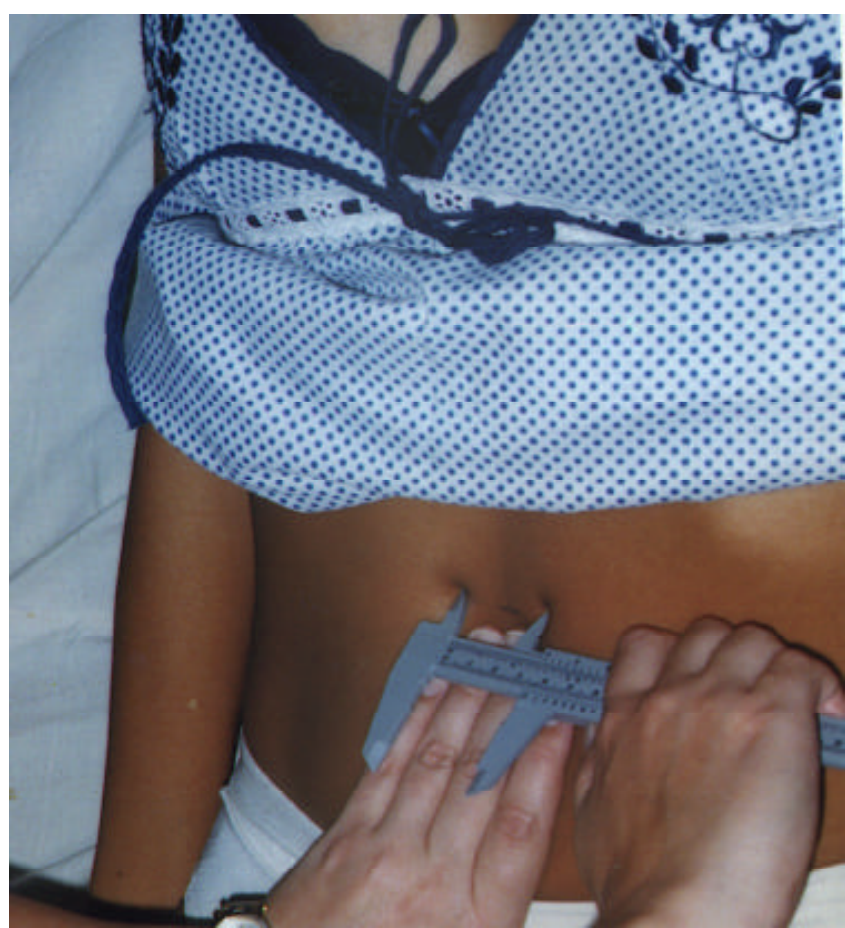

Figura 1 - Mensuração da diástase supra-umbilical dos músculos retos abdominais com 0 auxílio do paquímetro.

Todas as pacientes foram avaliadas, e no grupo de tratamento, as pacientes após avaliação se submeteram ao atendimento fisioterápico às 6 e 18 horas pós-parto, individualmente. No grupo controle foram realizadas duas avaliações, sendo a primeira 6 horas após o parto e a segunda 18 horas após o parto. Houve, portanto, um intervalo de 12 horas entre a $1^{\mathrm{a}}$ e $2^{\mathrm{a}}$ avaliação no grupo controle e entre o $1^{\circ}$ e $2^{\circ}$ atendimento para o grupo de tratamento. Este intervalo objetiva oferecer à puérpera um descanso entre uma e outra intervenção fisioterápica, assim como um tempo para o organismo se adaptar a esta intervenção. Os horários supracitados das avaliações e atendimentos foram determinados a partir da hora do parto de cada puérpera participante do estudo.

\section{Protocolo de atendimento}

A partir de uma posição inicial para todos os exercícios em decúbito dorsal, com flexão de quadris e joelhos e com os pés apoiados no leito, foram realizadas manobras de reeducação funcional respiratória, a partir de uma inspiração profunda da puérpera. Para tanto, a pesquisadora realizava manobras de alongamento diafragmático e bloqueio torácico até a percepção visual do padrão respiratório predominantemente abdominal. Em seguida, a puérpera inspirava profundamente, soltando o ar e contraindo a musculatura abdominal, por meio de uma expiração forçada.

Foram realizados também exercícios para estimulação dos músculos abdominais e pélvicos. 
Assim, em seqüência, com auxílio de um travesseiro entre os joelhos, a puérpera realizou um movimento de adução de quadris, associado à contração isométrica do assoalho pélvico. O próximo exercício constou de um movimento de retroversão pélvica, também associado à contração do assoalho pélvico mediante estímulo verbal da pesquisadora. Este foi seguido de exercício de contração isométrica dos músculos abdominais, principalmente o transverso abdominal. Neste exercício, a pesquisadora utilizouse do estímulo tátil, a sua própria mão, para obter a contração do andar inferior do abdome. Finalmente, realizou-se a contração isotônica dos músculos oblíquos abdominais, por meio do movimento de flexão anterior combinado com rotação do tronco, de modo que a borda inferior da escápula se retirasse do contato com o leito. Para facilitar a execução deste exercício, a puérpera era solicitada a colocar as mãos cruzadas sob a cabeça (região occipital), com abdução de ombros e flexão de cotovelos.

No primeiro atendimento, a puérpera realizou 10 repetições de cada exercício, e no segundo foram 20 repetições de cada exercício.

\section{Análise estatística}

Para a comparação entre as medidas da DMRA em cada um dos grupos, controle e tratamento, utilizou-se o teste não-paramétrico de Friedman. Para a comparação entre os grupos utilizou-se o teste $t$ de Student e o coeficiente de variância para determinar o nível de variação entre os grupos.

\section{Resultados}

\section{Caracterização da amostra}

A amostra de 50 puérperas, dividida entre o grupo controle $(\mathrm{N}=25)$ e o grupo de tratamento $(\mathrm{N}=25)$, foi caracterizada quanto a faixa etária, paridade e profissão.

A faixa etária das puérperas do estudo variou de 18 a 40 anos, havendo um predominio da faixa entre 18 e 25 anos nos dois grupos. Assim, o grupo controle contava com $40 \%$ das puérperas nesta idade, e o grupo de tratamento com $60 \%$ do total.

Com referência à paridade, no grupo controle $72 \%$ eram multíparas e $28 \%$ primíparas, e no grupo de tratamento havia $60 \%$ de multiparas e $40 \%$ de primiparas.

Relativo à profissão das puérperas, o grupo controle apresentou $80 \%$ de domésticas e do lar, $16 \%$ de balconistas e $4 \%$ de outras profissões. Já no grupo de tratamento, $80 \%$ eram domésticas e do lar, $12 \%$ recepcionistas e $8 \%$ de outras profissões.

A Tabela 1 mostra que houve diferença significativa $(p<0,0062)$ entre os grupos controle e tratamento com relação às medidas da DMRA supra-umbilical 6 horas pós-parto, em que o grupo tratamento apresentou uma média significativamente superior ao grupo controle. A pequena diferença encontrada no cálculo do coeficiente de variação $(\mathrm{CV})$ permite inferir que ambos os grupos apresentaram-se homogêneos antes de qualquer intervenção fisioterápica.

Tabela 1 - Comparação do percentual do valor das medidas da diástase supra-umbilical dos músculos retos abdominais 6 horas após o parto nos grupos controle e tratamento

\begin{tabular}{lllllll}
\hline \multicolumn{7}{c}{ Medidas descritivas (\% de redução) } \\
Grupo & Mínimo & Máximo & Média & DP & CV (\%) & p \\
\hline Controle & 30,0 & 42,0 & 31,6 & 2,6 & 8,23 & \\
Tratamento & 30,0 & 45,0 & 34,5 & 4,3 & 12,46 & \\
\hline
\end{tabular}

Nota: $O$ valor de $p$ (probabilidade de significância) na tabela refere-se ao teste $t$ de Student para amostras independentes; DP: desvio padrão; CV: coeficiente de variação.

Pode-se observar na Tabela 2 que existe diferença significativa entre as duas medidas da DMRA supra-umbilical no grupo controle ao longo do tempo $(\mathrm{p}<0,001)$, sendo que 6 horas após o parto ela foi significativamente superior à medida obtida 18 horas após o parto. O grupo controle apresenta uma discreta alteração do $\mathrm{CV}$, demonstrando uma homogeneidade dentro do grupo.

Tabela 2 - Medidas da diástase supra-umbilical dos músculos retos abdominais, em milímetros, do grupo controle $(\mathrm{N}=25)$ nos dois tempos avaliados

\begin{tabular}{lcccccc}
\hline \multicolumn{7}{c}{ Medidas descritivas (mm) } \\
$\begin{array}{l}\text { Tempo após } \\
\text { o parto }\end{array}$ & Mínimo & Máximo & Média & DP & CV (\%) & $p$ \\
\hline $6 \mathrm{~h}$ & 30,0 & 42,0 & 31,6 & 2,6 & 8,23 & \\
$18 \mathrm{~h}$ & 27,0 & 41,0 & 29,9 & 2,8 & 9,36 & \\
\hline
\end{tabular}

Nota: $O$ valor de $p$ (probabilidade de significância) na tabela refere-se ao teste $t$ de Student para amostras independentes; DP: desvio padrão; CV: coeficiente de variação.

Existe diferença significativa $(\mathrm{p}<0,001)$ entre as três medidas da DMRA supra-umbilical do grupo tratamento quanto aos tempos avaliados. Às 6 horas pós-parto ocorreu a maior média para a medida da DMRA supra-umbilical; após o primeiro atendimento e 18 horas pós-parto obteve-se a segunda maior medida, e após o segundo atendimento, a menor medida (Tabela 3). Com isso, pode-se dizer que houve uma redução das medidas da DMRA supra-umbilical ao longo do tempo. O CV mostra pequena oscilação nos três tempos, demonstrando que com o tratamento o grupo apresentou melhora do quadro (redução da DMRA) como um todo. 
Tabela 3 - Medidas da diástase supra-umbilical dos músculos retos abdominais, em milimetros, do grupo tratamento $(\mathrm{N}=25)$ nos três tempos avaliados.

\begin{tabular}{llllllll}
\hline \multicolumn{7}{c}{ Medidas descritivas $(\mathrm{mm})$} \\
Tempo & Mínimo & Máximo & Média & DP & CV $(\%)$ & $\mathrm{p}$ \\
\hline 6 h pós-parto & 30,0 & 45,0 & 34,5 & 4,3 & 12,46 & \\
Após $1^{\circ}$ atendimento & 22,0 & 41,0 & 30,1 & 4,6 & 15,28 & $<0,001$ \\
Após $2^{\circ}$ atendimento & 18,0 & 38,0 & 26,4 & 4,6 & 17,42 &
\end{tabular}

Nota: $O$ valor de $p$ (probabilidade de significância) na tabela refere-se ao teste $t$ de Student para amostras independentes; DP: desvio padrão; CV: coeficiente de variação.

As reduções percentuais das medidas da DMRA supra-umbilical de 6 horas pós-parto em relação à medida de 18 horas pós-parto no grupo controle e de 6 horas pós-parto em relação à medida após o segundo atendimento no grupo de tratamento foram significativamente diferentes $(p<0,001)$ (Tabela 4). Assim, o grupo controle apresentou uma média de redução de $5,4 \%$, que é inferior à obtida no grupo de tratamento, no qual a média de redução foi $12,5 \%$. Quanto ao CV, o grupo de tratamento ficou mais homogêneo após o atendimento fisioterápico do que o grupo controle, que se apresentou mais disperso. Nota-se, portanto, uma diferença de $17,8 \%$ de variação entre os dois grupos e entre seis e dezoito horas pós-parto.

Tabela 4 - Percentual de redução da medida da diástase supra-umbilical dos músculos retos abdominais nos grupos controle $(N=25)$ e tratamento $(N=25)$

\begin{tabular}{lllllll}
\hline \multicolumn{7}{c}{ Porcentagem de redução } \\
Grupo & Mínimo & Máximo & Média & DP & CV (\%) & p \\
\hline Controle & 0,0 & 10,0 & 5,4 & 2,3 & 42,6 & \\
Tratamento & 6,3 & 18,2 & 12,5 & 3,1 & 24,8 & \\
\hline
\end{tabular}

Nota: O valor de $\mathrm{P}$ (probabilidade de significância) na tabela refere-se ao teste $t$ de Student para amostras independentes; DP: desvio padrão; CV: coeficiente de variação.

\section{Discussão}

A escolha da mensuração da DMRA, no pós-parto imediato, está em concordância com a literatura ${ }^{2,3,5}$, que caracteriza a maior incidência desta diástase no terceiro trimestre de gestação e no pós-parto imediato. Em um estudo anterior ${ }^{3}$ esta diástase foi encontrada em toda a amostra, sendo que $62,5 \%$ apresentavam grau de separação superior a $3 \mathrm{~cm}$ no puerpério imediato. No presente estudo, a fim de minimizar esta condição de hipotonia e diminuir a DMRA, foram realizados estimulação proprioceptiva ${ }^{17}$, exercícios isométricos ${ }^{9}$ e isotônicos dos músculos abdominais (oblíquos, retos e transversos) e do assoalho pélvico ${ }^{8}$.
Em estudos anteriores $\mathrm{s}^{2,3,5}$, os critérios empregados na mensuração da DMRA eram subjetivos. Um instrumento confiável é necessário para conferir fidedignidade à mensuração desta diástase ${ }^{3}$ e assim verificar a redução da diástase objetivamente. Assim, neste estudo, um paquímetro foi originalmente empregado para este fim.

Para esta pesquisa, adotamos os critérios de Noble $^{12}$, em que uma diástase de dois dedos (mais ou menos $3 \mathrm{~cm}$ ) é considerada físiológica, não implicando, portanto, em complicações biomecânicas para o organismo materno. Este é o único critério descrito na literatura sobre a mensuração da DMRA. Foi possivel verificar que todas as medidas da diástase infra-umbilical foram inferiores a $3 \mathrm{~cm}$, o que permitiu excluí-las do estudo, a fim de preservar a coerência com os critérios de inclusão. Estes dados sobre a DMRA infra-umbilical estão em concordância com a disposição anatômica dos músculos retos abdominais em "V" no nivel em que se realiza esta medida ${ }^{11}$. A porção final dos músculos retos abdominais torna-se menos distensivel, porque neste ponto já há tecidos de tendão destes músculos ${ }^{7}$.

Boissonnault e Blaschak ${ }^{2}$ analisaram a incidência da DMRA em puérperas um ano após o parto e a partir daí iniciou-se um programa de exercícios de força para os músculos abdominais. No presente estudo objetivou-se a melhora da tonicidade da musculatura abdominal, uma vez que o protocolo de atendimento foi realizado dentro das primeiras 24 horas pós-parto. Numa fase mais avançada do puerpério (tardio e remoto) caberia a preocupação com o desenvolvimento de força dos músculos abdominais e pélvicos. Para tanto, é necessário um treinamento intensivo de pelo menos seis semanas para se obterem os primeiros indícios de desenvolvimento de força muscular ${ }^{1,10}$.

Os resultados obtidos neste estudo mostram uma diferença significativa $(\mathrm{p}<0,001)$ da porcentagem de redução da DMRA no pós-parto imediato entre grupos controle e tratamento. Este resultado não pode, no entanto, ser comparado com dados da literatura, pela escassez de investigação da influência de exercícios orientados para a puérpera neste período.

Conclui-se, então, que o atendimento fisioterápico no puerpério imediato contribuiu positivamente para a redução da DMRA mais precocemente. Esta pesquisa torna-se relevante por avaliar a redução da DMRA e por apresentar uma maneira nova de mensuração por meio do paquímetro, com parâmetros mais objetivos e quantitativos da medida desta diástase. 
SUMMARY

Purpose: to check if physiotherapy immediately after childbirth may contribute to early diastasis reduction.

Methods: longitudinal and randomized study with 50 parturient women recruited in the Maternidade-Escola Hilda Brandão of the Santa Casa of Belo Horizonte, from April to September 1998. The control group $(N=25)$ was submitted to evaluation and measurement ( $6 \mathrm{~h}$ and $18 \mathrm{~h}$ after labor), and the treatment group $(N=25)$ was submitted to the same evaluation and measurement as above, as well as to a protocol for physiotherapeutic assistance, 6 and $18 \mathrm{~h}$ after labor. For the evaluations, a pachymeter, a precision instrument, was used to measure diastasis.

Results: at 18 h after parturition, the control group presented a diastasis reduction of $5.4 \%$, and the treatment group of $12.5 \%$, as related to the first measure (6 $h$ after delivery) $(p<0.001$, with a confidence interval of $99 \%)$.

Conclusions: these results show that the physiotherapeutic assistance immediately after childbirth determines a significant reduction in the diastasis of the recti abdominis muscles (DRAM) after every treatment session, as well as a relevant reduction when compared to the control group, positively contributing to the earlier recovery of the recti abdominis muscles.

KEY WORDS: Diastasis recti abdominis. Puerperium. Physiotherapy. Pregnancy.

\section{Referências}

1. Artal R, Buckenmeyer P. Exercises during pregnancy and postpartum. Contemp OB/GYN 1995; 1: 3650 .

2. Boissonnault JS, Blaschak MJ. Incidence of diastasis recti abdominis during the childbearing year. Phys Ther 1988; 68: 1082-6.

3. Bursh SG. Interrater reliability of diastasis recti abdominis measurement. Phys Ther 1987; 67: 1077-9.

4. Ellis JW. Cuidados pós-parto. In: Ellis JW. Manual de Obstetrícia. 2.ed. Rio de Janeiro: Prentice-Hall do Brasil; 1986. p.75-9.

5. Gilleard LW, Brown JM. Structure and function of the abdominal muscles in primigravid subjects during pregnancy and the immediate postbirth period. Phys Ther 1996; 76: 750-62.

6. Gleeson PB, Pauls JA. Obstetrical physical therapy-review of the literature. Phys Ther 1988; 68: 1699-702.

7. Kapandji IA. A coluna lombar. In: Kapandji IA, editor. Fisiologia Articular: Esquemas Comentados de Mecânica Humana. 2.ed. São Paulo: Manole; 1987. v.3, p.96-107.

8. Konkler CJ. Principios de exercícios para a paciente obstétrica. In: Kisner C, Coldy LA, editores. Exercícios Terapêuticos: Fundamentos e Técnicas. 2.ed. São Paulo: Manole; 1996. p.547-76.

9. Lehmkuhl LD, Smith LK. Intervenção dos fatores mecânicos e fisiológicos na função muscular. In: Brunnstrom Cinesiologia Clinica. 4.ed. São Paulo: Manole; 1989. p.122-3.

10. Levy MN. Fisiologia do músculo esquelético. In: Berne RM, Levy MN, editores. Fisiologia. 3.ed. Rio de Janeiro: Guanabara Koogan; 1996. p.284-9.

11.Moore KL. O abdome. In: Moore KL, editor. Anatomia Orientada para Clínica. 3.ed. Rio de Janeiro: Guanabara Koogan; 1994. p.117-23.

12.Noble E. Essential exercises of the childbearing year. 2ed. Boston: Houghton Mifflin; 1982. p.58-63.

13.Polden M, Mantle J. Fisioterapia em ginecologia e obstetrícia. 1.ed. São Paulo: Livraria e Editora Santos, 1993.

14. Shirado O, Ito $T$, Kaneda $K$, Strase TE. Electromyographic analysis of four techniques for isometric trunk muscles exercises. Arch Phys Med Rehabil 1995; 76: 225-9.

15.Souza ELBL, Machado AV, Correa MD, Lapertosa L, Stanciolli LF, Almeida LC. Fisioterapia em obstetrícia e aspectos em neonatologia; uma visão multidisciplinar. 1.ed. Belo Horizonte: Editora Health, 1996.

16.Thornton SL, Thornton SJ. Management of gross divarication of the recti abdominis in pregnancy and labour. Physiotherapy 1993; 79: 457-8.

17.Voss DE, Ionta MKS, Myers BJ. Facilitação neuromuscular proprioceptiva: padrões e técnicas. 3.ed. São Paulo: Editora Médica Panamericana; 1990.

\section{Agradecimentos}

À Maternidade-Escola Hilda Brandão pela oportunidade, ao Comitê de Ética desta instituição pelo incentivo. Às professoras Elza L.B. Souza e Maria da Glória R. Machado pelo apoio. 